\title{
Fluorescence Fluctuation Spectroscopy in Subdiffraction Focal Volumes
}

\author{
Lars Kastrup, Hans Blom, Christian Eggeling, and Stefan W. Hell* \\ Department of NanoBiophotonics, Max Planck Institute for Biophysical Chemistry, 37077 Göttingen, Germany
}

(Received 26 October 2004; published 4 May 2005)

\begin{abstract}
We establish fluorescence fluctuation spectroscopy (FFS) with nanoscale detection volumes generated by stimulated emission depletion. Our method applies fluorescence correlation spectroscopy and fluorescence intensity distribution analysis to extract molecular information about mobilities and fluorescence emission in solution. The combination of correlation analysis with that of photon intensity distributions reveals a fivefold squeezing of the detection volume over current diffraction-limited systems, which is in full agreement with the simultaneously demonstrated 25-fold reduction in (axial) focal transit time. Our method significantly extends the potential of far-field FFS, including for the noninvasive investigation of molecular reactions at higher concentrations.
\end{abstract}

PACS numbers: 87.64. $-\mathrm{t}, 33.15 . \mathrm{Vb}, 66.10 . \mathrm{Cb}, 82.20 .-\mathrm{w}$

Movements and interactions of molecules are crucial in many chemical reactions as well as in most processes regulating life. Therefore, several methods have been developed to explore these molecular aspects in solution. Farfield fluorescence microscopy has become indispensable in this field, because by probing with micron-sized volumes of focused light, this noncontact approach leaves the molecular dynamics largely unperturbed. Moreover, with a single or only a few fluorescently tagged molecules in the volume at a time, fluctuations of fluorescence emission carry valuable information about molecular associations and reactions.

The first fluctuation analysis technique that has come into widespread use is fluorescence correlation spectroscopy (FCS) [1,2]. Whereas FCS registers the temporal structure of signal fluctuations, the more recent fluorescence intensity distribution analysis (FIDA) [3,4] gathers molecular information from the fluctuation amplitude. As these fluorescence fluctuation spectroscopy (FFS) tools have proven versatile, they have found an increasing number of users in biophysics and biochemistry [2].

When employing far-field optics, FFS has so far been restricted to using diffraction-limited focal volumes, that is, down to $\sim 0.1 \mathrm{fL}$ of minimal size. Consequently, to have only a few molecules in the focal volume, dilution to nanomolar concentrations is inevitable. Unfortunately, for many biological processes to work efficiently, micromolar concentrations are necessary. These limitations of FFS can be surmounted by reducing the focal volume, which is not straightforward because of diffraction. In theory, twophoton excitation squeezes the fluorescence spot by a factor of $\sqrt{2}$. In practice, however, requiring wavelength doubling, two-photon excitation rather increases the spot by $\sim \sqrt{2}$. The strength of two-photon FFS lies elsewhere [5,6].

Diffraction has, indeed, imposed a maximum molecular concentration that can be handled by far-field optical FFS. All efforts to reduce the detection volume any further implied the use of evanescent fields and mechanical constraints such as near-field optical tips [7], interfaces pro- viding total internal reflection [8,9], and waveguide structures [10,11]. However, mechanical confinement hampers noninvasive intracellular investigations, and the interaction with the probe is likely to affect the dynamics to be probed.

A different approach to overcome the diffraction barrier is to use stimulated emission depletion (STED) of the fluorescent molecular state $[12,13]$. STED is a far-field method that has displayed subdiffraction fluorescent focal volumes suitable for FFS $[13,14]$. An additional attractive feature of STED is that it allows one to adjust the detection volume. By increasing the power of the stimulating beam, STED can in principle scale down the spot to molecular dimensions. Employing STED, we now introduce fluorescence fluctuation spectroscopy with nanosized far-field detection volumes.

STED is best implemented with synchronized pulses. A pulse of $\sim 50 \mathrm{ps}$ duration for molecular excitation is swiftly followed by a $\sim 300$ ps STED pulse tuned towards the red tail of the emission spectrum of the dye. The STED pulse stimulates excited molecules down to a higher vibrational level of their ground state which further decays nonradiatively, usually within $<1 \mathrm{ps}$. The duration of the STED pulse is chosen such that the depletion is completed well within the typical excited state lifetime of several nanoseconds and, conversely, it is more than 100-fold longer than the $<1$ ps lifetime of the vibrational level into which the molecule is quenched. Thus, forcing the excited molecule into a quickly decaying state, the STED pulse quenches the fluorescence very effectively according to $F\left(h_{\mathrm{STED}}\right)=F_{0} \exp \left(-\sigma h_{\mathrm{STED}}\right)$ [13] where $F\left(h_{\mathrm{STED}}\right)$ is the remaining molecular fluorescence, $h_{\mathrm{STED}}$ (expressed in photons per $\mathrm{cm}^{2}$ per pulse) the local fluence of the STED beam, and $\sigma$ the molecular cross section for stimulated emission.

Spatial confinement of the fluorescence volume requires STED to be effected with a fluence featuring a zero at the focal center. A distribution $h_{\mathrm{STED}}(\vec{r})$ suitable for the spatial confinement of fluorescence is shown in Fig. 1(a). Referred 
to as the STED point spread function (PSF), $h_{\mathrm{STED}}(\vec{r})$ is realized through $\pi$-phase shifting the central half of the wave front of the STED beam incident into the objective's entrance pupil. Diffraction produces a STED PSF featuring two offset axial maxima, as well as a weaker ring-shaped maximum in the focal plane [13]. The subdiffraction dimensions of the fluorescence spot result from the exponential depletion of the excited state with the STED PSF. With $H_{0}(\vec{r})$ denoting the normalized probability of the molecule to be excited by the excitation pulse, the probability of emission is given by $H_{\text {spot }}(\vec{r})=H_{0}(\vec{r}) \exp \left[-\sigma h_{\mathrm{STED}}(\vec{r})\right]$. Electing the fluence at the maximum of the STED PSF $h_{\mathrm{STED}}^{\max } \gg \sigma^{-1}$ delivers a subdiffraction-sized and largely isotropic spot, because the fluorescence is confined to the central zero [Fig. 1(a)]. Profiles along all three axes were extracted from the calculated spots and fitted with Gaussians to deduce the $1 / e^{2}$ points, i.e., $w_{x, y}$ and $w_{z}$, along with the focal shape parameter $K=2 w_{z} /\left(w_{x}+w_{y}\right)$ [Fig. 1(b)].

The elongated spots of the customary diffraction-limited FFS demand the inclusion of shape parameters in the calculation of the fluorescence correlation function [2]

$$
G\left(t_{\mathrm{c}}\right)=1+\frac{1}{N}\left(\frac{1}{1+t_{\mathrm{c}} / \tau_{1}}\right) \sqrt{\frac{1}{1+t_{\mathrm{c}} / \tau_{2}}} .
$$

$\tau_{1}=\left[\left(w_{x}+w_{y}\right) / 2\right]^{2} / 4 D$ and $\tau_{2}=w_{z}^{2} / 4 D=K^{2} \tau_{1}$ represent the lateral and axial diffusion times through a Gaussian spot profile. $t_{\mathrm{c}}$ is the correlation lag time, $D$ is the diffusion coefficient, and $N$ is the apparent mean number of fluorescing molecules in the detection volume. The isotropic spot produced by STED implies $\tau_{1}=\tau_{2}$ which simplifies the FFS analysis. The exponential fluorescence suppression can render the spot profiles nonGaussian. However, calculations show that significant deviations from the Gaussian profile approximation occur only for high fluences and also predominantly in the direction perpendicular to the laser polarization, making Eq. (1) applicable.

In our experiment, the STED and excitation pulses were provided by a mode-locked Ti:sapphire laser $(785 \mathrm{~nm}$, $76 \mathrm{MHz}$, Coherent Mira, Santa Clara, CA) and a synchronized frequency-doubled optical parametric oscillator (585 nm, OPO, APE GmbH, Berlin, Germany), respectively. We measured $h_{\mathrm{STED}}(\vec{r})$ by probing the focal fluence with a scattering gold bead of subdiffraction diameter. This in turn allowed us to establish the fluence at the maxima of the STED PSF as $h_{\mathrm{STED}}^{\max }=1.6 \times 10^{19} \bar{P}_{\text {STED }} \mathrm{W}^{-1} \mathrm{~cm}^{-2}$, with $\bar{P}_{\text {STED }}$ denoting the time-averaged power of the STED pulse train transmitted by the lens. The fluorescence signal was spectrally separated from the laser light with bandpass filters and either processed by a hardware correlator (ALV6000, ALV GmbH, Langen, Germany) or registered by a single-photon counter (SPC-830, Becker\&Hickl $\mathrm{GmbH}$, Berlin, Germany) for fluctuation correlation and photon count histogram analysis, respectively.
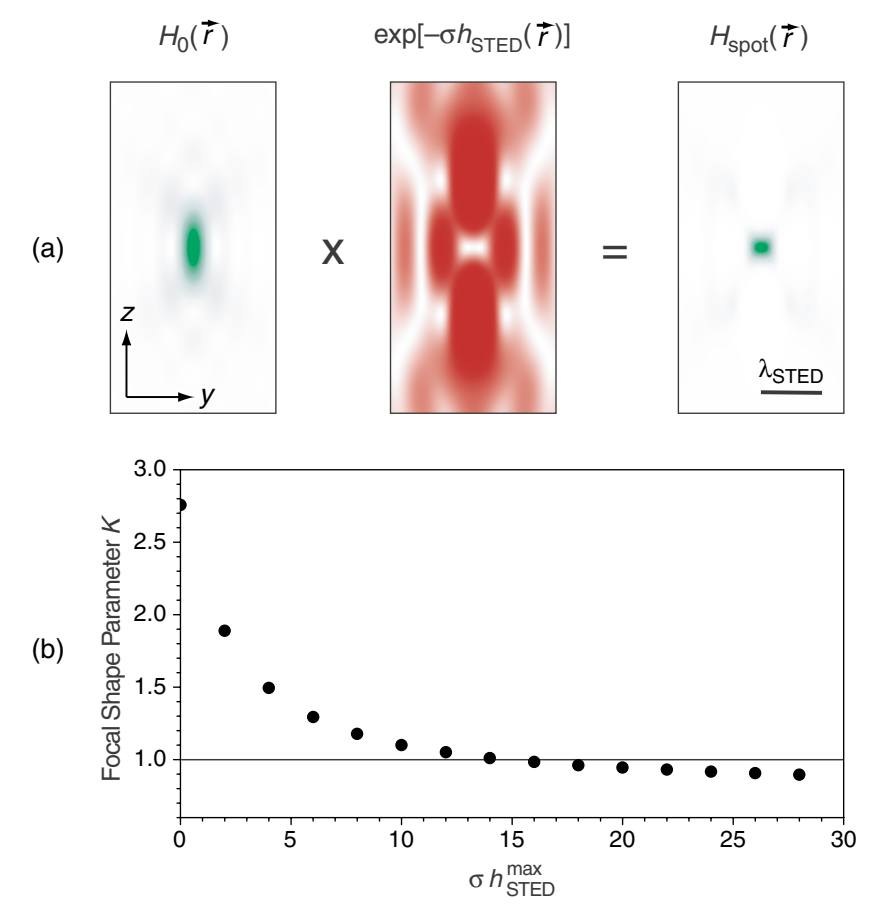

FIG. 1 (color online). (a) Diffraction-limited excitation spot $H_{0}(\vec{r})$ along with the STED PSF $h_{\mathrm{STED}}(\vec{r})$ and the resulting fluorescence spot $H_{\text {spot }}(\vec{r})$, calculated using vectorial diffraction theory. Conditions: $64^{\circ}$ semiaperture angle, water immersion, $x$-polarized excitation, and $\sigma h_{\mathrm{STED}}^{\max }=15$. (b) Focal shape parameter $K$ of the spot size with increasing STED fluence.

Figure 2(a) displays STED FCS traces for zero and maximal STED power acquired on a $\sim 50 \mathrm{nM}$ aqueous solution of the oxazine dye MR121. The shift of the autocorrelation curves towards shorter lag times with respect to the confocal reference indicates that the diffusional transit time through the focus becomes smaller when applying STED.

We have recorded a series of FCS curves with increasing STED power, but at the same dye concentration. The curves were analyzed by fitting them with Eq. (1). The attained count rates per molecule did not permit an independent determination of $\tau_{1}$ and $\tau_{2}$. Therefore, $\tau_{1}$ recorded at zero STED power was assumed constant. This is justified since $h_{\text {STED }}(\vec{r})$ did not really squeeze the focal volume in the lateral direction; see Fig. 1. Without STED, the focal volume is the usual confocal one with $\tau_{1} \approx 25 \mu \mathrm{s}$ and $\tau_{2} \approx 480 \mu \mathrm{s}$, respectively, giving a focal shape parameter $K$ of $\sim 4$, which is a typical value attained in practice. With increasing STED beam power, the fitted axial diffusion time decreases to $\sim 20 \mu \mathrm{s}$ [Fig. 2(b)], proving that we have achieved a spherical fluorescence spot and thus reduced the axial diffusion time 25 -fold. Because $V \propto \sqrt{\tau_{2}}$, this implies a fivefold reduction of the volume $V$. Despite the fivefold narrowing along the optic axis, the observed shift of the autocorrelation curve is only by a factor of 1.5. This is in accordance with Eq. (1), because in a far-field microscope the axial diffusion contributes less to the fluctuations as compared to that along the lateral directions. 

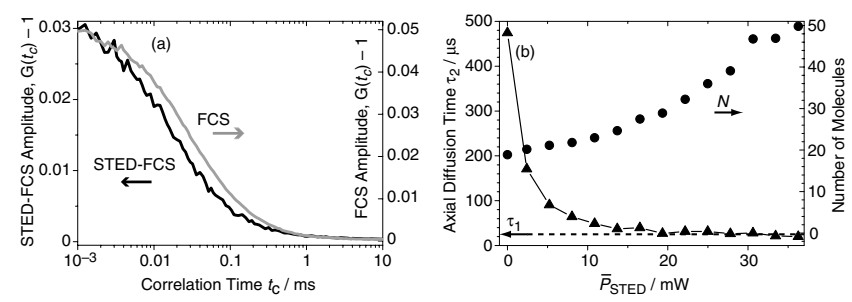

FIG. 2. (a) Measured FCS curves with and without a timeaveraged STED power of $\bar{P}_{\text {STED }}=22 \mathrm{~mW}$. (b) Deduced axial diffusion time $\tau_{2}$ (triangles) and apparent mean number of focal spot molecules $N$ (circles) as a function of $\bar{P}_{\mathrm{STED}}$.

The apparent mean number of fluorescent molecules $N$ resulting from the standard FCS evaluation surprisingly increases with increasing $\bar{P}_{\text {STED }}$ [Fig. 2(b)], which is in contradiction to the expectation $N \propto V$. Therefore, we evaluated the fluctuations by FIDA, which discloses the numbers of molecules of different species with different brightnesses along with potential uncorrelated signal contributions. The analysis is based on the photon count histogram $\tilde{P}(n)$ set up from the number of counts $n$ detected within consecutive time intervals of equal width $T$. The theoretical model for $\tilde{P}(n)$ is a convolution of the photon count probability distributions $P(n)$ for all spatial elements $d V$ which make up the detection volume. Each $P(n)$ is characterized by a double Poissonian distribution of the number of molecules $m$ as well as of the photon counts $n$ [4]

$$
\begin{aligned}
P(n)= & \frac{(U T)^{n}}{n !} e^{-U T}+\sum_{m}\left\{\frac{(c d V)^{m}}{m !} e^{-c d V}\right\} \\
& \times\left\{\frac{\left(m Q H_{\text {spot }}(\vec{r}) T\right)^{n}}{n !} e^{-m Q H_{\text {spot }}(\vec{r}) T}\right\} .
\end{aligned}
$$

$N^{\prime}=c d V$ describes the mean number of fluorescent molecules in the volume element $d V, Q$ is their mean specific brightness, and $U$ accounts for contributions of uncorrelated signal components. The convolution of contributions from different volume elements $d V$ is carried out in the Fourier domain, using an empirically parametrized $H_{\text {spot }}(\vec{r})$ [4]. The numerical modeling of $\tilde{P}(n)$ yields $N^{\prime}, Q$, and $U$.

The measurements described in Fig. 2 were essentially repeated to build up the FIDA histograms $(T=40 \mu \mathrm{s})$ which were fitted using the FCS++ ANALYZE software package (Evotec Technologies, Hamburg, Germany). The number of molecules $N^{\prime}$ deduced from FIDA [Fig. 3(a)] shows a fivefold decrease at the highest STED power applied, which agrees with the volume reduction deduced from the 25 -fold reduction in axial diffusion time. We note that the value of $N^{\prime}$ is biased towards higher values by a small constant factor. This stems from the fact that the bin width is slightly larger than the mean focal transit time of the molecule of $\sim 25 \mu \mathrm{s}$ [4].

Figure 3(b) displays the uncorrelated signal component $U$ along with the total count rate $S$ extracted using FIDA, showing an onset of $U$ and an associated decrease of $S$ with increasing $\bar{P}_{\text {STED }}$. The occurrence of $U$ can be explained only by incomplete STED, which is present in this fluorophore solution even at high $\bar{P}_{\text {STED }}$. Indeed, it is found that, with this dye-wavelength combination, STED leaves a fluorescent offset $\delta$ [Fig. 3(c)]. The effect on the spot shape expected for incomplete STED is illustrated in Fig. 3(d) displaying the calculated axial profiles of normalized fluorescence emission. Molecules diffusing through the focal periphery therefore give rise to some fluorescence, which is only weakly correlated with that from the sharp central spot. We have excluded that $U$ originates from the laser beams or from ambient light. This observation is important, since the total laser power deposited in the focal region is increased in STED FFS. $U$ notably follows a similar saturation behavior as the measured STED depletion. Moreover, time-correlated single-photon counting revealed the same fluorescence lifetime $(1.8 \mathrm{~ns})$ of the signal $U$ as the dye in use.

With $U$ established by FIDA, the amplitude of the FCS curves determined by Eq. (1) can now be corrected for the uncorrelated signal using $\tilde{N}=N(S-U)^{2} / S^{2}$ [2]. $N$ and $\tilde{N}$ are, respectively, the apparent and the corrected number of molecules in the focal volume. With this correction, the initial increase in the apparent number of focal molecules in the FCS data is converted into the expected fivefold decrease in the true number of molecules $\tilde{N}$ (Fig. 4, squares). The decrease is in accordance with the similarly normalized number gained by FIDA alone, and with the normalized volume confinement determined from the axial diffusion times (FCS). The resulting ratios agree in their dependence on $\bar{P}_{\text {STED }}$ (Fig. 4), independently further confirming the central volume reduction down to $20 \mathrm{aL}$.
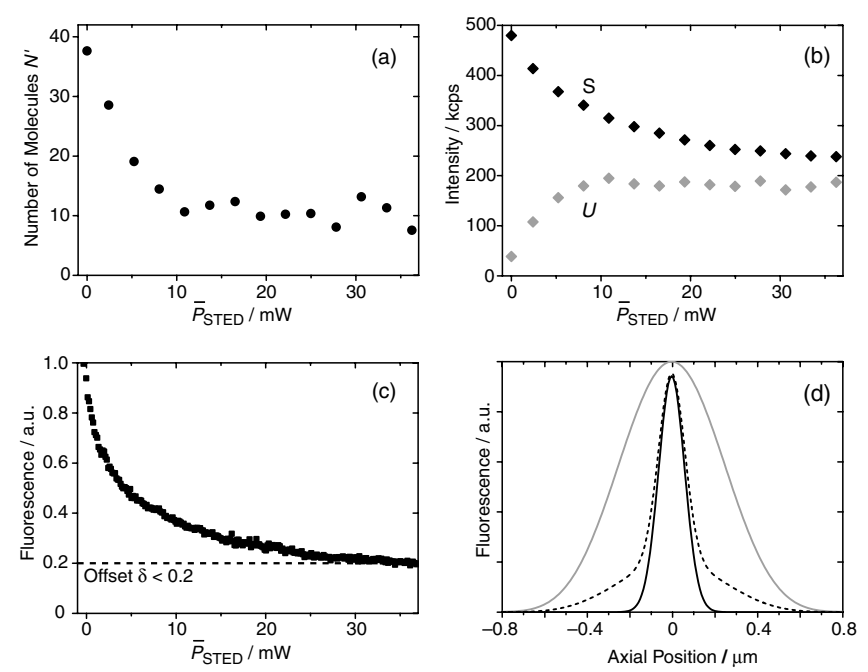

FIG. 3. (a) Number of molecules $N^{\prime}$. (b) Total signal $S$ and uncorrelated signal component $U$ as extracted by FIDA. (c) Experimental fluorescence suppression through STED. (d) Calculated axial fluorescence profile without (gray) and with STED at $\sigma h_{\mathrm{STED}}^{\max }=30$ (black and dashed lines corresponding to $\delta=0$ and 0.2 , respectively). 


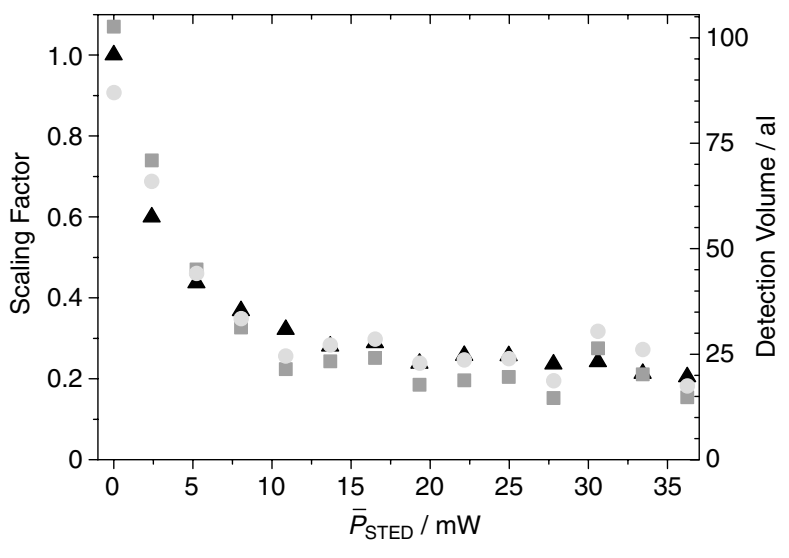

FIG. 4. Scaling of the detection volume as a function of $\bar{P}_{\text {STED }}$ determined from (triangles) the axial diffusion time $\tau_{2}$ gained by FCS, (squares) the corrected number of molecules $\tilde{N}$ from the FCS amplitude, and (circles) the number of molecules $N^{\prime}$ from FIDA alone. Right axis shows the detection volume in attoliters $\left(10^{-18} \mathrm{~L}\right)$ obtained according to $V=8 \pi^{3 / 2} D \tau_{1} \sqrt{D \tau_{2}}$ and using $D=2.5 \times 10^{-6} \mathrm{~cm}^{2} / \mathrm{s}$.

As can be inferred from Fig. 3(d), the narrowed fluorescence spot is expected to deviate from a Gaussian shape with increasing $\bar{P}_{\text {STED }}$ due to the undepleted fluorescence offset. While we have, indeed, noticed a slight decrease in the goodness of the fit to the correlation data at the largest powers applied, the Gaussian approximation was sufficient for this study. At high STED powers $\left(\bar{P}_{\text {STED }}>30 \mathrm{~mW}\right)$ a more elaborate fit is advisable if accurate quantitative results are to be obtained.

Although two-photon excitation would not reduce the effective spot in absolute values, it may contribute to reducing some of the background fluctuations arising from more remote points in the focal region. We also note in this context that an advantage of two-photon over single-photon excitation confocal FCS is that contributions from the secondary diffraction maxima of excitation are lower, which simplifies Gaussian fits to initially nonGaussian excitation spots [15].

Contrary to an imaging application, a slight bleaching by the STED beam is not strictly adverse in STED FFS because, contributing to the reduction of the effective focal volume, bleaching in the spot periphery may work to the advantage of the method. The associated apparent reduction of the concentration can be considered by gauging with a standard solution. While in a number of dyes, the application of STED involves a slight accentuation of photobleaching, the aqueous solution of MR121 proved remarkably photostable. In fact, we have not observed appreciable photobleaching in connection with STED.

The necessity of the analysis by FIDA is ascribed to the offset $\delta$ displayed in the curve of Fig. 3(c) resulting from incomplete fluorescence suppression. Reasons for $\delta$ are that in some dyes the subnanosecond STED pulses may induce non-negligible (multiphoton) excitation of ground state molecules. Another issue is that STED is weighted by the orientation of the molecular transition dipole with respect to the stimulating field. This is not critical if the rotational molecular motion is much faster or much slower than the duration of the STED pulse, but otherwise also a cause for incomplete suppression. Furthermore, transient dark states of the molecules may also interfere with fluorescence depletion. Finally, a nonzero central minimum of $h_{\mathrm{STED}}(\vec{r})$ weakens fluorescence in the center and hence limits the signal to background ratio [Fig. 3(d)]. This limitation can be counteracted by using adjustable phase modulation in the STED PSF creation.

We note, however, that neither the exponential depletion nor any other assumption about STED entered our FFS analysis. In fact, the actual depletion behavior, Fig. 3(c), is not critical to the results obtained. Even with incomplete depletion, the combined STED FFS analysis extends fluorescence fluctuation spectroscopy to subdiffraction volumes. Since there is no theoretical limit to the size that can be created, refinements of STED should confine the fluorescent spot even further, eventually enabling the study of molecular dynamics at very high concentrations $[13,14]$.

We thank A. Schönle for help with the measurement software. This work was supported by the Volkswagen Foundation.

*Electronic address: hell@ nanoscopy.de

[1] D. Magde, E. L. Elson, and W. W. Webb, Phys. Rev. Lett. 29, 705 (1972).

[2] R. Rigler and E.S. Elson, Fluorescence Correlation Spectroscopy. Theory and Applications (Springer-Verlag, Berlin, Heidelberg, 2001).

[3] Y. Chen, J. D. Müller, P. T. C. So, and E. Gratton, Biophys. J. 77, 553 (1999).

[4] P. Kask, K. Palo, D. Ullmann, and K. Gall, Proc. Natl. Acad. Sci. U.S.A. 96, 13756 (1999).

[5] W. Denk, J. H. Strickler, and W. W. Webb, Science 248, 73 (1990).

[6] K. M. Berland, P. T. So, and E. Gratton, Biophys. J. 68, 694 (1995).

[7] E. Betzig, J. K. Trautmann, T. D. Harris, J. S. Weiner, and R. L. Kostelak, Science 251, 1468 (1991).

[8] N.L. Thompson, T.P. Burghardt, and D. Axelrod, Biophys. J. 33, 435 (1981).

[9] T. Ruckstuhl and S. Seeger, Opt. Lett. 29, 569 (2004).

[10] M. J. Levene, J. Korlach, S. W. Turner, M. Foquet, H. G. Craighead, and W. W. Webb, Science 299, 682 (2003).

[11] M. Foquet, J. Korlach, W. R. Zipfel, W. W. Webb, and H. G. Craighead, Anal. Chem. 76, 1618 (2004).

[12] S. W. Hell and J. Wichmann, Opt. Lett. 19, 780 (1994).

[13] T. A. Klar, S. Jakobs, M. Dyba, A. Egner, and S. W. Hell, Proc. Natl. Acad. Sci. U.S.A. 97, 8206 (2000).

[14] T. A. Laurence and S. Weiss, Science 299, 667 (2003).

[15] S. T. Hess and W. W. Webb, Biophys. J. 83, 2300 (2002). 